\title{
The role of expansin and xyloglucan endotransglycosylase genes in the regulation of plant growth under changing environmental conditions
}

Kuluev B.R.*, Mikhaylova E.V., Knyazev A.V., Berezhneva Z.A. Institute of Biochemistry and Genetics, UFRC RAS, Ufa, Russia

*e-mail:kuluev@bk.ru

The discrepancy of cellulose microfibrils during cell expansion is achieved by three basic mechanisms: hydrolysis of a part of binding glycans with endoglycanases, cutting and new crosslinking of glycans with xyloglucan endo-transglycosylases/hydrolases (XTHs), and the violation of hydrogen bonds between the microfibrils of cellulose and glycan chains, which is carried out by expansins. We have created transgenic tobacco plants with increased expression of the genes encoding the tobacco expansins: NtEXPA1, $N t E X P A 5$ and tomato xyloglucan endo-transglycosylase - $t X E T-B 2$. Increased expression of NtEXPA1 and NtEXPA5 expansin genes led to an increase in the growth rate and root length both under normal plant growth conditions and at $12{ }^{\circ} \mathrm{C}$ and $50 \mathrm{mM} \mathrm{NaCl}$. Increased expression of expansin genes influenced the changes in the fresh and dry mass of a shoot, leading to an increase in their exposure to hypothermia. Overexpression of the $t X E T-B 2$ gene promoted tobacco root growth in a medium containing $50 \mathrm{mM} \mathrm{NaCl}$. Under drought conditions, overexpression of $t X E T-B 2$ gene resulted in a considerable increase in fresh and dry weight in many of the studied transgenic lines. The totality of the obtained data may indicate the involvement of NtEXPA1, NtEXPA5, and $t X E T-B 2$ genes in the regulation of growth under hypothermia, drought, and salinity. We will be used these target genes for the genetic transformation of cultivated plants.

Acknowledgements: This work was supported by Russian Foundation for Basic Research, grant No. 18-04-00118A. 